\title{
Most patients with a first episode of major depressive disorder experienced multiple levels of depressive symptoms over time
}

\author{
Judd LL, Akiskal HS, Maser JD, et al. A prospective 12-year study of subsyndromal and syndromal depressive symptoms in unipolar \\ major depressive disorders. Arch Gen Psychiatry 1998 Aug;55:694-700.
}

\section{Question}

Do patients with major depressive disorder have multiple levels of depressive symptoms and frequent changes in symptom levels over time?

\section{Design}

Inception cohort of patients followed for up to 12 years (mean follow up 8.5 y) (National Institute of Mental Health Collaborative Depression Study).

\section{Setting}

5 tertiary care centres in the US.

\section{Patients}

431 patients were enrolled in the study, of whom $122(28 \%$, mean age 39 y, 52\% women)* had a first episode of major depressive disorder without dysthymia according to the Research Diagnostic Criteria derived from the Schedule for Affective Disorders and Schizophrenia; spoke English; were white; had an IQ score $\geqslant 70$; and had no evidence of organic mental disorder, terminal medical illness, bipolar disorder, schizoaffective disorder, or schizophrenia.

\section{Assessment of prognostic factors}

Interviews were used to assess depressive symptoms at baseline.

\section{Main outcome measure}

The number of weeks spent at 4 levels of symptom severity was assessed weekly by using the Psychiatric Status Rating Scales, which were linked to the Research Diagnostic Criteria. Weekly symptom severity ratings were collected by using chronological memory prompts during interviews every 6 months for the first 5 years and yearly thereafter. Interviews were supplemented by detailed review of clinical, medical, and research records for the first 5 years. Patients were classified as having no depressive symptoms; subsyndromal depressive symptoms; minor or intermittent depressive or dysthymic disorder symptoms; or major depressive disorder symptoms.

\section{Main results}

During a mean follow up of 8.5 years, patients with a first episode of major depression at baseline had no symptoms 54\% of the time, subsyndromal depressive symptoms $14 \%$ of the time, minor depressive disorder symptoms $21 \%$ of the time, and major depressive disorder symptoms $12 \%$ of the time. Among patients with $\geqslant 2$ years of follow up (92\%), $14 \%$ had no asymptomatic weeks, $61 \%$ had symptoms at all 4 severity levels, $26 \%$ had symptoms at 3 severity levels, 11\% had symptoms at 2 severity levels, and 3\% had symptoms at 1 severity level. Patients had a mean change of 1.5 changes/year (range 0 to 7 changes/year) and a mean total of 13 changes (range 1 to 80 changes) in symptom severity levels.

\section{Conclusion}

Most patients with a first episode of major depressive disorder had depressive symptoms at 3 or 4 levels of severity over time.

*In accordance with our selection criteria for prognosis studies, (see p 2-3), we have only included data for 122 first episode patients in this abstract.

Sources of funding: in part, National Institute of Mental Health and the Roehr Fund of the University of California, San Diego.

For correspondence:Dr L L Judd,Department of Psychiatry, University of California, San Diego, 9500 Gilman Drive, La Jolla, CA 92093-0603, USA. Fax +16195347653.

\section{Commentary}

This important study by Judd et al addresses the question of whether various levels of severity of depressive symptomatology represent levels of severity of a single disorder or multiple distinct clinical entities.

This study was done in a tertiary care setting and thus its findings may not be generalisable to primary or secondary care settings. Treatment received by study patients was presumably superior to that received by the average patient because study patients were treated in major university medical centres. Patients whose depressive disorders are untreated or who receive poorer quality care may experience worse long term outcomes.

The finding that the same patient typically moves through 3 or 4 various levels of symptom severity during a follow up period suggests that the various severity levels represent levels of a single disorder. Additional studies showing a common biological or psychological pathway or underpinning to the various symptom levels are needed to validate this conclusion.

A major finding was that patients with a first episode of major depressive disorder had symptoms of depression $46 \%$ of the time over an average of 8.5 years of follow up. This is a clinically significant finding and has important implications for the quality of life of these patients. Clinicians would do well to inform these patients and their families that they have a disorder more like hypertension (a chronic disorder) than like pneumonia (an acute disorder).

The finding that depressed patients experience varying levels of symptoma- tology over long periods of time suggests that it is important that clinicians work with patients to identify stressors or other vulnerability factors that may precipitate exacerbation of depressive symptoms, to promote lifestyle changes (eg, reduce substance use, increase exercise) that might reduce risk of depression, and to clarify decision criteria about when to return to active treatment. ${ }^{1}$

Jacqueline B Persons, PhD University of California, San Francisco and Center for Cognitive Therapy Oakland, California, USA

\footnotetext{
1 Burbach FR. The efficacy of physical activity interventions within mental health services: Anxiety and depressive disorders. I Mental
} Health (UK) 1997;6:543-66. 